\title{
WELL POSEDNESS AND CONTROL OF SEMILINEAR WAVE EQUATIONS WITH ITERATED LOGARITHMS*
}

\author{
Piermarco Cannarsa ${ }^{1}$, Vilmos Komornik ${ }^{2}$ and Paola Loreti ${ }^{3}$
}

\begin{abstract}
Motivated by a classical work of Erdős we give rather precise necessary and sufficient growth conditions on the nonlinearity in a semilinear wave equation in order to have global existence for all initial data. Then we improve some former exact controllability theorems of Imanuvilov and Zuazua.
\end{abstract}

Résumé. Motivé par un travail classique d'Erdős on donne des conditions nécessaires et suffisantes de croissance de la non linéarité dans une équation des ondes semilinéaire pour l'existence des solutions globales pour toutes les données initiales. Ensuite on améliore certains théorèmes antérieurs de contrôlabilité exacte de Imanuvilov et de Zuazua.

AMS Subject Classification. 35L05, 35L70, 34A40.

Received October 5, 1998. Revised February 3, 1999.

\section{INTRODUCTION AND FORMULATION OF THE MAIN RESULTS}

Consider the problem

$$
\left\{\begin{array}{l}
u^{\prime \prime}-\Delta u-f(u)=0 \text { in } \Omega \times(0, T), \\
u=0 \text { on } \Gamma \times(0, T), \\
u(0)=u_{0} \text { and } u^{\prime}(0)=u_{1} \text { in } \Omega,
\end{array}\right.
$$

where

- $\Omega$ is a nonempty bounded open domain in $\mathbb{R}^{N}$ having a boundary $\Gamma$ of class $C^{2}$;

Keywords and phrases: Wave equation, semilinear equation, integral inequality

* Most of this work was done during the visit of the first author at the Université Louis Pasteur in Strasbourg, the visits of the second author at the Università di Roma "Tor Vergata", the Istituto per le Applicazioni del Calcolo "Mauro Picone" del CNR, the Università degli Studi di Roma "La Sapienza", and the visits of the three authors at the Institut Henri Poincaré in Paris in the framework of a special trimester devoted to the Control Theory and Its Applications (in January 1998). They thank these institutions for their hospitality.

1 Dipartimento di Matematica, Università di Roma "Tor Vergata", Via della Ricerca Scientifica, 00133 Roma, Italy; e-mail: cannarsa@axp.mat.uniroma2.it

2 Institut de Recherche Mathématique Avancée, Université Louis Pasteur et CNRS, 7 rue René Descartes, 67084 Strasbourg Cedex, France; e-mail: komornik@math.u-strasbg.fr

${ }^{3}$ Istituto per le Applicazioni del Calcolo "Mauro Picone", Consiglio Nazionale delle Ricerche, Viale del Policlinico 137, 00161 Roma, Italy. Current address: Dipartimento MeMoMat, Università di Roma "La Sapienza", Via A. Scarpa 16, 00161 Roma, Italy. e-mail: loreti@dmmm.uniroma1.it

(c) EDP Sciences, SMAI 1999 
- $f: \mathbb{R} \rightarrow \mathbb{R}$ is a given function of class $C^{1}$;

- $T$ is a given positive number.

For the existence of a unique maximal solution

$$
u \in C\left(\left[0, T^{\prime}\right] ; H_{0}^{1}(\Omega)\right) \cap C^{1}\left(\left[0, T^{\prime}\right] ; L^{2}(\Omega)\right), \quad 0<T^{\prime} \leq T,
$$

in case $N \geq 2$ we need the additional property

$$
\begin{cases}\left|f^{\prime}(s)\right| \leq c\left(1+|s|^{2 /(N-2)}\right) & \text { if } N \geq 3 \\ \left|f^{\prime}(s)\right| \leq c\left(1+|s|^{\alpha}\right) & \text { for some finite } \alpha \text { if } N=2\end{cases}
$$

for all $s \in \mathbb{R}$. No condition of this type is needed if $N=1$. We refer, e.g., to [2], Chapter 6, for proof.

First we study the existence of a (unique) global solution

$$
u \in C\left([0, T] ; H_{0}^{1}(\Omega)\right) \cap C^{1}\left([0, T] ; L^{2}(\Omega)\right)
$$

for every given

$$
u_{0} \in H_{0}^{1}(\Omega) \quad \text { and } \quad u_{1} \in L^{2}(\Omega)
$$

without any sign condition on $f$. We need another growth assumption on $f$. For this we introduce the primitive $F$ of $f$ defined by

$$
F(s)=\int_{0}^{s} f(z) d z, \quad s \in \mathbb{R} .
$$

Furthermore, motivated by [3], let us introduce the iterated logarithm functions $\log _{j}$ defined by the formulae

$$
\log _{0} s:=s \quad \text { and } \quad \log _{j} s:=\log \left(\log _{j-1} s\right), \quad j=1,2, \ldots
$$

Define $e_{j}$ by the equation $\log _{j} e_{j}=2$.

Theorem 1.1. Let $f: \mathbb{R} \rightarrow \mathbb{R}$ be a function of class $C^{1}$. Assume (1.2) if $N \geq 2$. Furthermore, assume that there exist a positive integer $k$ and a number $\beta>0$ such that

$$
|F(s)| \leq \beta \prod_{j=0}^{k} \log _{j}^{2}|s| \text { for all }|s|>e_{k} .
$$

Then for every $u_{0}, u_{1}$ satisfying (1.4) the problem (1.1) has a unique global solution satisfying (1.3).

Our proof will show that the quantity

$$
\int_{\Omega}\left(u^{\prime}(t)\right)^{2}+|\nabla u(t)|^{2}-2 F(u(t)) d x
$$

does not depend on $t \in[0, T]$.

We will also show that the condition (1.5) is essentially optimal.

Theorem 1.2. Assume that there exist a positive integer $k$, a real number $p>2$ and two positive numbers $\alpha_{0}$ and $c$ such that

$$
F(s) \geq \alpha_{0}\left(\prod_{j=0}^{k-1} \log _{j}^{2} s\right) \log _{k}^{p} s \text { for all } s>c .
$$


Then there exist initial data

$$
u_{0} \in H_{0}^{1}(\Omega) \quad \text { and } \quad u_{1} \in L^{2}(\Omega)
$$

such that (1.1) has no global solution satisfying (1.3).

Remarks.

- Theorem 1.1 was first proved by Cazenave and Haraux [1] in the case where $f$ is a constant multiple of the function $s \log s$. They considered the case $\Omega=\mathbb{R}^{N}$, but their method equally works for bounded domains.

- Subsequently Zuazua [10] noticed that their method also applies under the weaker assumption (1.5) for $k=1$, and he also proved Theorem 1.2 for $k=1$.

- One can readily verify that if

$$
f(s)=O\left(|s| \prod_{j=1}^{k} \log _{j}^{2}|s|\right) \quad \text { as } \quad|s| \rightarrow \infty
$$

then condition (1.5) is satisfied.

- Zuazua asked whether the condition (1.5) can be weakened by replacing the product by an infinite linear combination of the products indexed by $k$, with a suitable sequence of coefficients converging sufficiently quickly to zero. In order to not make this paper too long, we shall study this question in a later work.

- If there exist a positive integer $k$, a real number $p>2$ and two positive numbers $\alpha_{1}$ and $c_{1}$ such that

$$
f(s) \geq \alpha_{1} s\left(\prod_{j=1}^{k-1} \log _{j}^{2} s\right) \log _{k}^{p} s \text { for all } s>c_{1},
$$

then condition (1.6) is satisfied. Indeed, we have

$$
F(s)=\int_{0}^{s} f(t) d t \geq \int_{s / 2}^{s} f(t) d t \geq \alpha_{1} \frac{s}{2} \frac{s}{2}\left(\prod_{j=1}^{k-1} \log _{j}^{2} \frac{s}{2}\right) \log _{k}^{p} \frac{s}{2} .
$$

If $s$ is sufficiently large, say $s>c$, then

$$
\log _{j} \frac{s}{2}>\frac{\log _{j} s}{2} \text { for } j=1, \ldots, k
$$

and therefore

$$
F(s) \geq \alpha_{1} 2^{-2-2(k-1)-p}\left(\prod_{j=0}^{k-1} \log _{j}^{2} s\right) \log _{k}^{p} s,
$$

i.e. (1.6) is satisfied with

$$
\alpha_{0}=\alpha_{1} 2^{-2-2(k-1)-p} .
$$

Next we study the boundary controllability of the system

$$
\left\{\begin{array}{l}
u^{\prime \prime}-\Delta u-f(u)=0 \text { in } \Omega \times(0, T), \\
u=h \text { on } \Gamma \times(0, T), \\
u(0)=u_{0} \text { and } u^{\prime}(0)=u_{1} \text { in } \Omega .
\end{array}\right.
$$


We only consider the one-dimensional case

$$
\Omega=(a, b)
$$

so we rewrite it in the following form:

$$
\left\{\begin{array}{l}
u_{t t}-u_{x x}-f(u)=0 \quad \text { in }(a, b) \times(0, T), \\
u(t, a)=h_{a}(t) \text { and } u(t, b)=h_{b}(t) \text { for } t \in(0, T), \\
u(0, x)=u_{0}(x) \text { and } u_{t}(0, x)=u_{1}(x) \text { for } \quad x \in(a, b)
\end{array}\right.
$$

Definition. The problem (1.7) is exactly controllable at time $T$ if for every

$$
\left(u_{0}, u_{1}\right),\left(v_{0}, v_{1}\right) \in H^{1}(\Omega) \times L^{2}(\Omega)
$$

there exist control functions

$$
h_{a}, h_{b} \in H^{1}(0, T)
$$

such that (1.7) has a global solution satisfying the final conditions

$$
u(T)=v_{0} \quad \text { and } \quad u^{\prime}(T)=v_{1} \quad \text { in } \quad \Omega .
$$

Theorem 1.3. Assume that $f$ satisfies the growth condition (1.5) for some positive integer $k$. If

$$
T>b-a,
$$

then the problem (1.7) is exactly controllable at time $T$.

Remarks.

- This theorem answers in particular a question of Zuazua [10]. He proved the exact controllability of (1.1) by assuming instead of (1.5) that

$$
|F(s)|<\beta_{0} s^{2} \log ^{2}|s| \text { for all }|s|>c
$$

for a sufficiently small $\beta_{0}$ and for a sufficiently large $c>0$, and he asked whether the same conclusion holds for large $\beta_{0}$. Our theorem provides, in particular, an affirmative answer to such a question. Note, however, that Zuazua also obtained analogous results by controlling at only one endpoint of the interval $(a, b)$, and for a related internal controllability problem.

- The optimality of condition (1.8) follows from the finite propagation speed for the wave equation (at least if $f \equiv 0)$.

To prove Theorem 1.1 we shall apply the approach of Cazenave and Haraux [1]. In particular, we shall need a general nonlinear version of Gronwall's inequality (see Th. 2.1 below), which may be interesting itself.

The proof of Theorem 1.2 will use the ideas of Zuazua [10] by showing that the solution blows up before $T$.

We were unable to adapt Zuazua's fixed point method for the proof of Theorem 1.3. Instead, we apply a former method of Imanuvilov [4], based on characteristics.

The authors are grateful to O. Imanuvilov for his many helpful explications concerning his former work [4] and for providing them with the detailed proof of the trace regularity property in Proposition 5.1. Furthermore, they thank the referee and E. Zuazua for several helpful remarks and suggestions. 


\section{Gronwall type inequalities AND iterated LOGARIthmS}

Theorem 2.1. Let $g:[0, \infty) \rightarrow \mathbb{R}$ be a continuous and non-decreasing function. Assume that there exists a positive number $c$ such that

$$
g>0 \text { in }(c, \infty) \quad \text { and } \quad \int_{c}^{\infty} \frac{1}{g(s)} d s=\infty
$$

Let $\varphi:[0, T] \rightarrow \mathbb{R}$ be a continuous, nonnegative function, satisfying for some numbers $A, B>0$ the inequalities

$$
\varphi(t) \leq A+B \int_{0}^{t} g(\varphi(s)) d s \quad \text { for all } t \in[0, T] .
$$

Then $\varphi$ is bounded on $[0, T]$ by a constant depending only on $g, A, B$ and on $T$.

Remarks.

1) More precisely, putting

$$
G(t):=\int_{c}^{t} \frac{1}{g(s)} d s, \quad t \in(0, \infty)
$$

we have

$$
\varphi(t) \leq G^{-1}(G(A)+B t), \quad \text { for all } t \in[0, T] .
$$

2) The original Gronwall lemma corresponds to the choice $g(s)=s$.

3) Cazenave and Haraux [1] considered the case $g(s)=s \log (1+s)$.

4) We shall use later the more general case where

$$
g(s)=\left(e_{k}+s\right) \log \left(e_{k}+s\right) \ldots \log _{k}\left(e_{k}+s\right)=\prod_{j=0}^{k} \log _{j}\left(e_{k}+s\right)
$$

for some positive integer $k$ and with $e_{k}$ as defined above by $\log _{k} e_{k}=2$.

Proof of Theorem 2.1. It follows from our assumptions that $G$ is of class $C^{1}$, strictly increasing, and it maps the interval $(0, \infty)$ onto $(G(0+), \infty)$. We have

$$
\frac{d}{d t}\left[G\left(A+B \int_{0}^{t} g(\varphi(s)) d s\right)\right]=G^{\prime}\left(A+B \int_{0}^{t} g(\varphi(s)) d s\right) B g(\varphi(t))=B \frac{g(\varphi(t))}{g\left(A+B \int_{0}^{t} g(\varphi(s)) d s\right)} \leq B .
$$

Therefore

$$
G(\varphi(t)) \leq G\left(A+B \int_{0}^{t} g(\varphi(s)) d s\right) \leq G(A)+B t
$$

and hence

$$
\varphi(t) \leq G^{-1}(G(A)+B t), \quad t \in[0, T]
$$

as claimed. 
Now we generalize a crucial technical lemma in [1] to iterated logarithms. For this we introduce the iterated exponential functions $\exp _{j}: \mathbb{R} \rightarrow \mathbb{R}$ by the formulas

$$
\exp _{0} x=x \quad \text { and } \quad \exp _{j} x=\exp \left(\exp _{j-1} x\right), j=1,2, \ldots
$$

Given an integer $k \geq 0$, set $e_{k}=\exp _{k} 2$ and

$$
L_{k}(x)=\prod_{j=0}^{k} \log _{j}\left(e_{j}+|x|\right), \quad x \in \mathbb{R} .
$$

This function is well defined, even, and increasing for $x \geq 0$. Furthermore, we deduce from the inequality

$$
L_{k}(x) \geq(2+|x|) \prod_{j=1}^{k} \log _{j} e_{j}=2^{k}(2+|x|) \geq 2^{k+1}>0
$$

that

$$
L_{k}(x) \geq|x| \quad \text { and } \quad L_{k}(x) \geq 2>0
$$

for all $x$ and $k$. We shall use these properties several times.

In order to simplify the notations henceforth we denote by $\|\cdot\|_{p}$ the usual norm of $L^{p}(\Omega)$ and we write simply $\|\cdot\|$ instead of $\|\cdot\|_{2}$.

We need a generalization of a lemma in [1].

Lemma 2.2. Given $k \geq 0$ and $\varepsilon>0$ arbitrarily, there is a constant $c(\varepsilon)$ such that

$$
\left\|L_{k}(u)\right\| \leq \varepsilon\|\nabla u\|+c(\varepsilon) L_{k}(\|u\|)
$$

for all $u \in H_{0}^{1}(\Omega)$, where $\|\cdot\|$ denotes the usual norm in $L^{2}(\Omega)$.

Proof. Assume for simplicity that $N \geq 3$ : the cases $N=1,2$ are analogous and simpler. We recall that by the Sobolev imbedding theorem there exists a constant $S$ such that

$$
\|u\|_{2 N /(N-2)}^{2} \leq S\|\nabla u\|^{2}
$$

for all $u \in H_{0}^{1}(\Omega)$.

Given $\delta>0$ arbitrarily, there exists a constant $c(\delta)>0$ such that

$$
L_{k}(x)^{2} \leq \delta|x|^{(2 N+4) / N}+c(\delta)
$$

for all real $x$. Since

$$
\frac{N}{2 N+4}=\alpha \frac{1}{2}+(1-\alpha) \frac{N-2}{2 N}
$$

if $\alpha=2 /(N+2)$, applying the interpolational inequality we have (denoting by $|\Omega|$ the volume of $\Omega$ )

$$
\begin{aligned}
\left\|L_{k}(u)\right\|^{2} & \leq \delta\|u\|_{(2 N+4) / N}^{(2 N+4) / N}+c(\delta)|\Omega| \leq \delta\left(\|u\|^{2 /(N+2)}\|u\|_{2 N /(N-2)}^{N /(N+2)}\right)^{(2 N+4) / N}+c(\delta)|\Omega|=\delta\|u\|^{4 / N}\|u\|_{2 N /(N-2)}^{2} \\
& +c(\delta)|\Omega| \leq \delta S\|u\|^{4 / N}\|\nabla u\|^{2}+c(\delta)|\Omega| .
\end{aligned}
$$


Since $L_{k}>1$ everywhere, in case $\|u\| \leq 1$ hence we deduce the estimate

$$
\left\|L_{k}(u)\right\|^{2} \leq \delta S\|\nabla u\|^{2}+c(\delta)|\Omega| L_{k}(\|u\|)^{2},
$$

and (2.3) follows by choosing $\delta=S^{-1} \varepsilon^{2}$.

Henceforth assume that $\|u\|>1$. Let us note that

$$
\log (c+a b) \leq \log (c+a)+\log (c+b)
$$

for all $a, b \geq 0$ and $c \geq 1$, and that

$$
\log (a+b) \leq \log a+\log b
$$

for all $a, b \geq 2$. Hence for every $a, b \geq 0$ we have the inequalities

$$
\begin{aligned}
\log _{j}\left(e_{j}+a b\right) & \leq \log _{j-1}\left[\log \left(e_{j}+a\right)+\log \left(e_{j}+b\right)\right] \leq \log _{j-2}\left[\log _{2}\left(e_{j}+a\right)+\log _{2}\left(e_{j}+b\right)\right] \\
& \leq \cdots \leq 2 \max \left\{\log _{j}\left(e_{j}+a\right), \log _{j}\left(e_{j}+b\right)\right\}
\end{aligned}
$$

for $j=1,2, \ldots, k$. Distinguishing the cases $a \geq b$ and $a<b$ hence we deduce the inequality

$$
\begin{aligned}
\left(\prod_{j=1}^{k} \log _{j}\left(e_{j}+a b\right)\right)^{2} & \leq 4^{k} \max \left\{\left(\prod_{j=1}^{k} \log _{j}\left(e_{j}+a\right)\right)^{2},\left(\prod_{j=1}^{k} \log _{j}\left(e_{j}+b\right)\right)^{2}\right\} \\
& \leq 4^{k}\left(\prod_{j=1}^{k} \log _{j}\left(e_{j}+a\right)\right)^{2}+4^{k}\left(\prod_{j=1}^{k} \log _{j}\left(e_{j}+b\right)\right)^{2}
\end{aligned}
$$

Now given $u \in H_{0}^{1}(\Omega)$ such that $\|u\|>1$, setting $v:=u /\|u\|$ and applying (2.5) we have

$$
\begin{aligned}
\int_{\Omega} L_{k}(u)^{2} d x= & \int_{|u| \leq\|u\|} L_{k}(u)^{2} d x+\int_{|u|>\|u\|} L_{k}(u)^{2} d x \leq|\Omega| L_{k}(\|u\|)^{2} \\
& +\int_{|u|>\|u\|}(2+|u|)^{2}\left(\prod_{j=1}^{k} \log _{j}\left(e_{j}+|u|\right)\right)^{2} d x \leq|\Omega| L_{k}(\|u\|)^{2} \\
& +4^{k} \int_{|u|>\|u\|}(2+|u|)^{2}\left(\prod_{j=1}^{k} \log _{j}\left(e_{j}+|v|\right)\right)^{2} d x \\
& +4^{k} \int_{|u|>\|u\|}(2+|u|)^{2}\left(\prod_{j=1}^{k} \log _{j}\left(e_{j}+\|u\|\right)\right)^{2} d x=:|\Omega| L_{k}(\|u\|)^{2}+I_{1}+I_{2} .
\end{aligned}
$$

Since $\|u\|>1$ implies

$$
2+|u| \leq\|u\|(2+|v|),
$$

we have

$$
I_{1} \leq 4^{k} \int_{|u|>\|u\|}\|u\|^{2} L_{k}(v)^{2} d x \leq 4^{k}\|u\|^{2}\left\|L_{k}(v)\right\|^{2} .
$$


Furthermore, since $|u|>\|u\|>1$ implies

$$
2+|u| \leq 3|u|
$$

we have

$$
I_{2} \leq 9 \cdot 4^{k} \int_{|u|>\|u\|}|u|^{2} d x\left(\prod_{j=1}^{k} \log _{j}\left(e_{j}+\|u\|\right)\right)^{2} \leq 9 \cdot 4^{k} L_{k}(\|u\|)^{2} .
$$

Substituting this into the above inequality we find that

$$
\left\|L_{k}(u)\right\|^{2} \leq|\Omega| L_{k}(\|u\|)^{2}+4^{k}\|u\|^{2}\left\|L_{k}(v)\right\|^{2}+9 \cdot 4^{k} L_{k}(\|u\|)^{2} .
$$

Applying (2.4) for $v$ and using the inequality $L_{k}(x) \geq|x|$ hence we obtain that

$$
\begin{aligned}
\left\|L_{k}(u)\right\|^{2} & \leq\left\{|\Omega|+9 \cdot 4^{k}\right\} L_{k}(\|u\|)^{2}+4^{k}\|u\|^{2} \delta S\|\nabla v\|^{2}+4^{k}\|u\|^{2} c(\delta)|\Omega| L_{k}(1)^{2} \\
& \leq 4^{k} \delta S\|\nabla u\|^{2}+\left\{|\Omega|+4^{k} c(\delta)|\Omega| L_{k}(1)^{2}+9 \cdot 4^{k}\right\} L_{k}(\|u\|)^{2} .
\end{aligned}
$$

Choosing $\delta=4^{-k} S^{-1} \varepsilon^{2}$ the lemma follows.

\section{Proof of Theorem 1.1}

According to the general Theorem 6.1.4 in [8], it suffices to show that the maximal solution cannot blow up before $t=T$. Thus Theorem 1.1 will follow from the

Lemma 3.1. Consider the unique maximal solution of (1.1), defined on some interval $\left[0, T^{\prime}\right)$ with $0<T^{\prime} \leq T$. Then

$$
u \in L^{\infty}\left(0, T^{\prime} ; H_{0}^{1}(\Omega)\right), \quad u^{\prime} \in L^{\infty}\left(0, T^{\prime} ; L^{2}(\Omega)\right), \quad F(u) \in L^{\infty}\left(0, T^{\prime} ; L^{1}(\Omega)\right) .
$$

Proof. Multiplying the equation in (3.1) by $u^{\prime}$ and integrating by parts we obtain that the quantity

$$
\int_{\Omega}\left(u^{\prime}(t)\right)^{2}+|\nabla u(t)|^{2}-2 F(u(t)) d x
$$

is conserved. Applying Lemma 2.2 we obtain that

$$
\left\|u^{\prime}\right\|^{2}+\|\nabla u\|^{2} \leq c+\left\|L_{k}(u)\right\|^{2} \leq c+\varepsilon\|\nabla u\|^{2}+c(\varepsilon) L_{k}(\|u\|)^{2} .
$$

Choosing $\varepsilon=1$ and using the estimate

$$
\|u(t)\| \leq\left\|u_{0}\right\|+\int_{0}^{t}\left\|u^{\prime}(s)\right\| d s=:\left\|u_{0}\right\|+\varphi(t)
$$

it follows that

$$
\varphi^{\prime}(t)=\left\|u^{\prime}(t)\right\| \leq c+c(1) L_{k}\left(\left\|u_{0}\right\|+\varphi(t)\right)
$$

and therefore

$$
\varphi(t) \leq c T+c \int_{0}^{t} L_{k}\left(\left\|u_{0}\right\|+\varphi(s)\right) d s, \quad 0 \leq t<T^{\prime} .
$$


Now choose $k^{\prime}>k$ such that

$$
L_{k}\left(\left\|u_{0}\right\|+x\right) \leq L_{k^{\prime}}(x)
$$

for all $x \geq 0$, and apply Theorem 2.1 with

$$
g(s)=L_{k}\left(\left\|u_{0}\right\|+s\right) .
$$

We obtain that

$$
\varphi \in L^{\infty}\left(0, T^{\prime}\right)
$$

Using (3.3) it follows that

$$
u \in L^{\infty}\left(0, T^{\prime} ; L^{2}(\Omega)\right)
$$

and then, applying (3.2) again, this time with $\varepsilon<1$, we conclude that

$$
u \in L^{\infty}\left(0, T^{\prime} ; H_{0}^{1}(\Omega)\right)
$$

and

$$
u^{\prime} \in L^{\infty}\left(0, T^{\prime} ; L^{2}(\Omega)\right) .
$$

Finally, by the conservation of the quantity (3.1) we have

$$
F(u) \in L^{\infty}\left(0, T^{\prime} ; L^{1}(\Omega)\right),
$$

as stated.

\section{Proof of Theorem 1.2}

It is sufficient to prove Theorem 1.2 for small $T$ 's. Set

$$
x_{0}=\frac{a+b}{2}
$$

and fix

$$
0<T<R<\frac{b-a}{2}
$$

arbitrarily. Thanks to (1.5) we have

$$
\int_{c}^{\infty} \frac{d s}{\sqrt{F(s)}} \leq c_{1} \int_{c}^{\infty} \frac{d s}{s \log s \ldots \log _{k-1} s\left(\log _{k} s\right)^{p / 2}}=c_{1}\left(1-\frac{p}{2}\right)^{-1}\left[\left(\log _{k} s\right)^{1-\frac{p}{2}}\right]_{c}^{\infty}=\frac{2 c_{1}}{p-2}\left(\log _{k} c\right)^{1-\frac{p}{2}}<\infty .
$$

Therefore we can choose a positive constant $\alpha>c$ such that

$$
\int_{\alpha}^{\infty} \frac{d s}{\sqrt{F(s)}}<\sqrt{2} T
$$


and choose $u_{0} \in H_{0}^{1}(\Omega)$ such that

$$
u_{0}=\alpha \text { in }\left(x_{0}-R, x_{0}+R\right) .
$$

Furthermore, choose another positive constant $\beta$, and choose $u_{1} \in L^{2}(\Omega)$ such that

$$
u_{1}=\beta \text { in }\left(x_{0}-R, x_{0}+R\right) \text {. }
$$

We claim that the corresponding solution of (1.1) blows up before $T$.

Assume on the contrary that $u$ is defined in $(a, b) \times(0, T)$. It follows from the finite propagation property that for any fixed $x \in\left(x_{0}-R+T, x_{0}+R-T\right)$, the function $v(t):=u(x, t)$ satisfies the ordinary differential equation

$$
v^{\prime \prime}-f(v)=0 \quad \text { in } \quad(0, T)
$$

with the initial conditions

$$
v(0)=\alpha \quad \text { and } \quad v^{\prime}(0)=\beta \text {. }
$$

(In particular, it does not depend on the particular choice of $x$.)

It follows from the differential equation that the quantity

$$
E(t):=\left|v^{\prime}(t)\right|^{2}-2 F(v(t))
$$

is in fact independent of $t \in(0, T)$. Hence

$$
v^{\prime}(t)=\sqrt{E(0)+2 F(v(t))}
$$

and therefore

$$
T=\int_{\alpha}^{v(T)} \frac{d v}{\sqrt{E(0)+2 F(v)}} \leq \int_{\alpha}^{\infty} \frac{d v}{\sqrt{2 F(v)}}
$$

But this is impossible because the last integral is less than $T$ by the choice of $\alpha$.

\section{Proof of Theorem 1.3}

We apply a method of Imanuvilov [4]. First we deduce from Theorem 1.1 an auxiliary result on the Cauchy problem in a triangle. Given a bounded interval $(-d, d)$, set

$$
Q=\left\{(x, t) \in \mathbb{R}^{2}:-d<x<d, 0<t<d-|x|\right\}
$$

and

$$
S=\left\{(x, t) \in \mathbb{R}^{2}:-d<x<d, t=d-|x|\right\}
$$

Then $Q$ is a triangle with basis $(-d, d)$ and altitude $d$, and $S$ is the union of the other two sides; see Figure 1 .

Consider the problem

$$
\left\{\begin{array}{l}
u_{t t}-u_{x x}-f(u)=0 \quad \text { in } \quad Q \\
u(0, x)=u_{0}(x) \text { and } u_{t}(0, x)=u_{1}(x) \text { for } \quad x \in(-d, d) .
\end{array}\right.
$$




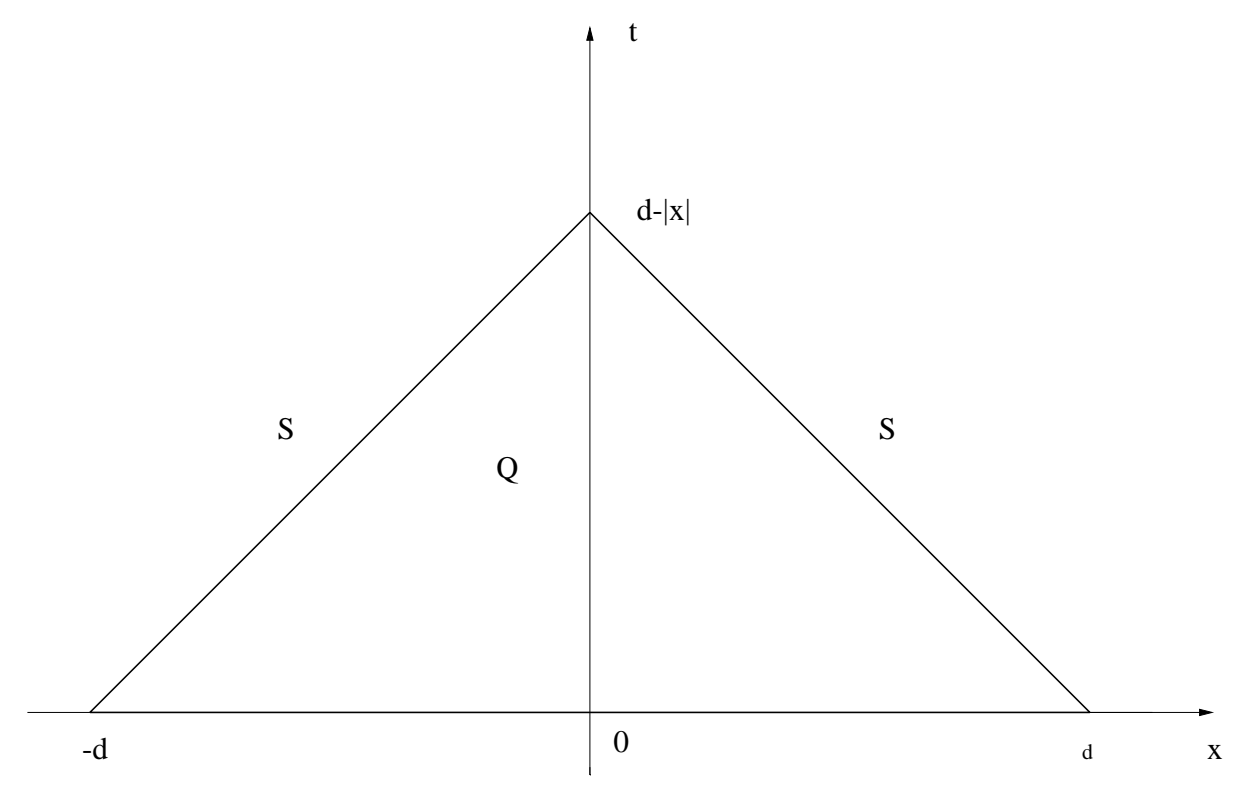

FigURE 1

Proposition 5.1. Assume that $f$ satisfies for some positive integer $k$ the condition (1.5), and let

$$
u_{0} \in H^{1}(-d, d), \quad u_{1} \in L^{2}(-d, d) .
$$

Then the problem (5.1) has a unique solution $u \in H^{1}(Q)$ for which the traces $u_{t}(\cdot, t), u_{x}(\cdot, t)$ are well defined in $L^{2}(t-d, d-t)$ for every $0 \leq t \leq d$, and the function

$$
t \mapsto \int_{t-d}^{d-t} u_{t}^{2}+u_{x}^{2} d x
$$

is continuous (hence bounded) on $[0, d]$.

Furthermore, the trace of the solution on $S$ satisfies

$$
\left.u\right|_{S} \in H^{1}(S) .
$$

Remark.

Note that the above properties imply that solutions are bounded: $u \in L^{\infty}(Q)$.

Proof. Let us choose a bounded interval $\Omega=(a, b)$ containing $[-d, d]$ in its interior, and extend $u_{0}$ and $u_{1}$ to $\Omega$ such that

$$
u_{0} \in H_{0}^{1}(a, b), \quad u_{1} \in L^{2}(a, b) .
$$

Then by Theorem 1.1 the problem (1.1) has a (unique) global solution

$$
\bar{u} \in C\left([0, d] ; H^{1}(a, b)\right) \cap C^{1}\left([0, d] ; L^{2}(a, b)\right) .
$$

Its restriction $u$ to $Q$ has the desired properties. 
Let us note that if $f$ is globally Lipschitz continuous and if the initial data are more regular:

$$
u_{0} \in H^{2}(\Omega) \cap H_{0}^{1}(\Omega) \quad \text { and } \quad u_{1} \in H_{0}^{1}(\Omega),
$$

then by standard regularity results we have

$$
\bar{u} \in C\left([0, T] ; H^{2}(\Omega) \cap H_{0}^{1}(\Omega)\right) \cap C^{1}\left([0, T] ; H_{0}^{1}(\Omega)\right) \cap C^{2}\left([0, T] ; L^{2}(\Omega)\right) .
$$

Its restriction $u$ to $Q$ will be called a strong solution.

For the proof of the uniqueness, let $u^{1}, u^{2}$ be two solutions and set $w=u^{1}-u^{2}$, so that

$$
\left\{\begin{array}{l}
w_{t t}-w_{x x}=\varphi w \text { in } Q, \\
w(0, x)=w_{t}(0, x)=0 \text { for } \quad x \in(-d, d)
\end{array}\right.
$$

where

$$
\varphi=\int_{0}^{1} f^{\prime}\left(\lambda u^{1}+(1-\lambda) u^{2}\right) d \lambda \in L^{\infty}(Q) .
$$

Therefore

$$
w(t, x)=\frac{1}{2} \int_{0}^{t} \int_{x-(t-\tau)}^{x+(t-\tau)} \varphi w d \sigma d \tau
$$

Define

$$
W(t)=\sup _{|x|<d-t}|w(t, x)|, \quad 0 \leq t \leq d,
$$

then

$$
W(t) \leq\|\varphi\|_{\infty} \int_{0}^{t} W(\tau) d \tau, \quad 0 \leq t \leq d
$$

and so $W \equiv 0$ by the usual Gronwall lemma.

For the proof of (5.2), set

$$
E(t)=\int_{t-d}^{d-t} u_{t}^{2}+u_{x}^{2} d x, \quad 0 \leq t \leq d .
$$

Since the solution is bounded, modifying $f$ outside a sufficiently large interval we may assume that $f$ is globally Lipschitz continuous. Then for every strong solution (see the definition above) we have

$$
\begin{aligned}
E^{\prime}(t) & =-\left(u_{t}^{2}+u_{x}^{2}\right)(t, d-t)-\left(u_{t}^{2}+u_{x}^{2}\right)(t, t-d)+\int_{t-d}^{d-t} 2 u_{t} u_{t t}+2 u_{x} u_{x t} d x \\
& =-\left(u_{t}-u_{x}\right)^{2}(t, d-t)-\left(u_{t}+u_{x}\right)^{2}(t, t-d)+\int_{t-d}^{d-t} 2 u_{t}\left(u_{t t}-u_{x x}\right) d x \\
& =-2 u_{\tau}^{2}(t, d-t)-2 u_{\tau}^{2}(t, t-d)+\int_{t-d}^{d-t} 2 f(u) u_{t} d x
\end{aligned}
$$


where $u_{\tau}$ denotes the tangential derivative along $S$. Since $E(d)=0$, integrating we obtain the identity

$$
\sqrt{2} \int_{S} u_{\tau}^{2} d S=E(0)+\int_{Q} F(u) d x .
$$

This identity remains valid for all solutions by an approximation argument.

Since the right-hand side of the identity (5.3) is finite, the property (5.2) follows.

Remark. Let us note for further reference that the proof of identity (5.3) remains valid for every function $u \in C^{2}(Q) \cap C^{2}(\bar{Q})$ satisfying

$$
u_{t t}-u_{x x}-f(u)=0 \text { in } Q .
$$

Now, using the same notation as in Proposition 5.1, consider the Goursat problem

$$
\left\{\begin{array}{l}
u_{t t}-u_{x x}-f(u)=0 \quad \text { in } \quad Q \\
u=\psi \text { on } S .
\end{array}\right.
$$

Proposition 5.2. Assume that $f$ satisfies condition (1.5) for some positive integer $k$, and let $\psi \in H^{1}(S)$. Then the problem (5.4) has a unique solution $u \in H^{1}(Q)$ such that the traces $u_{t}(\cdot, t), u_{x}(\cdot, t)$ are well defined in $L^{2}(t-d, d-t)$ for every $0 \leq t \leq d$, and the function

$$
t \mapsto \int_{t-d}^{d-t} u_{t}^{2}+u_{x}^{2} d x
$$

is continuous (hence bounded) on $[0, d]$.

Proof. Uniqueness. We note that, as in the preceding proposition, solutions belong to $L^{\infty}(Q)$. If $u^{1}, u^{2}$ solve (5.4), then $w:=u^{1}-u^{2}$ satisfies

$$
\left\{\begin{array}{l}
w_{t t}-w_{x x}=\varphi w \text { in } Q \\
w=0 \text { on } S
\end{array}\right.
$$

where

$$
\varphi=\int_{0}^{1} f^{\prime}\left(\lambda u^{1}+(1-\lambda) u^{2}\right) d \lambda \in L^{\infty}(Q) .
$$

We have to show that $w$ vanishes identically. More generally, we will show that if a function $w$, having the same regularity as the solutions in the formulation of the proposition, satisfies

$$
\left\{\begin{array}{l}
w_{t t}-w_{x x}=\varphi w \text { in } Q \\
w=\psi \text { on } S
\end{array}\right.
$$

for some $\varphi \in L^{\infty}(Q)$ and $\psi \in H^{1}(S)$, then

$$
\|w\|_{H^{1}(Q)}+\left\|w_{t}\right\|_{L^{2}\left(0, d ; L^{2}(t-d, d-t)\right)}+\left\|w_{x}\right\|_{L^{2}\left(0, d ; L^{2}(t-d, d-t)\right)} \leq c^{\prime}\|\psi\|_{H^{1}(S)}
$$

with a constant $c^{\prime}$ depending only on $R$ if $\|\varphi\|_{L^{\infty}(Q)} \leq R$.

First, from the inequalities

$$
|w(x, t)| \leq|w(x, t)-w(t-d, t)|+|\psi(t-d, t)|
$$


and

$$
|w(x, t)-w(t-d, t)| \leq\left\|w_{x}\right\|_{L^{1}(t-d, d-t)} \leq c\left\|w_{x}\right\|_{L^{2}(t-d, d-t)}
$$

we deduce the estimate

$$
\|w(\cdot, t)\|_{L^{2}(t-d, d-t)} \leq c\left\|w_{x}\right\|_{L^{2}(t-d, d-t)}+c|\psi(t-d, t)|
$$

Next, setting

$$
E(t)=\int_{t-d}^{d-t} w_{t}^{2}+w_{x}^{2} d x
$$

and repeating the computation of the preceding proof, we obtain

$$
E^{\prime}(t)=-2 \psi_{\tau}^{2}(t, d-t)-2 \psi_{\tau}^{2}(t, t-d)+\int_{t-d}^{d-t} 2 \varphi w w_{t} d x
$$

Since $E(d)=0$, it follows that

$$
\begin{aligned}
E(t)= & 2 \int_{t}^{d} \psi_{\tau}^{2}(s, d-s)+\psi_{\tau}^{2}(s, t-s) d s-\int_{t}^{d} \int_{s-d}^{d-s} 2\left(\varphi w w_{t}\right)(x, s) d x d s \\
& \leq \sqrt{2}\left\|\psi_{\tau}\right\|_{L^{2}(S)}^{2}+\int_{t}^{d} \int_{s-d}^{d-s} w^{2}(x, s)+\|\varphi\|_{L^{\infty}(Q)}^{2} w_{t}^{2}(x, s) d x d s .
\end{aligned}
$$

Hence, using (5.6), we deduce that

$$
\begin{aligned}
E(t) & \leq \sqrt{2}\left\|\psi_{\tau}\right\|_{L^{2}(S)}^{2}+c \int_{t}^{d} \psi^{2}(s-d, s) d s+\int_{t}^{d} \int_{s-d}^{d-s} c w_{x}^{2}(x, s)+\|\varphi\|_{L^{\infty}(Q)}^{2} w_{t}^{2}(x, s) d x d s \\
& \leq c\|\psi\|_{H^{1}(S)}^{2}+c\left(1+\|\varphi\|_{L^{\infty}(Q)}^{2}\right) \int_{t}^{d} E(s) d s .
\end{aligned}
$$

Applying the usual Gronwall lemma, the above inequality yields

$$
\sup _{0 \leq t \leq d} E(t) \leq c^{\prime}\|\psi\|_{H^{1}(S)}^{2}
$$

with a constant $c^{\prime}$ depending only on $R$ if $\|\varphi\|_{L^{\infty}(Q)} \leq R$. Since (5.6) implies that

$$
\|w\|_{L^{2}(Q)}^{2} \leq c^{\prime}\left(\|\psi\|_{H^{1}(S)}^{2}+\sup _{0 \leq t \leq d} E(t)\right)
$$

the desired inequality (5.5) follows from (5.7) and (5.8).

Existence. Assume first that $f$ is globally Lipschitz continuous. Then for every $\psi \in C^{1}(S)$ the problem $(5.4)$ has a solution $u \in C^{1}(\bar{Q})$, defined in a suitable weak sense. Indeed, the proof given in [9] (lecture 5) for the linear case remains valid for the globally Lipschitz case.

Now, given $\psi \in H^{1}(S)$ arbitrarily, choose a sequence $\left(\psi^{n}\right)$ in $H^{1}(S)$ such that the corresponding solutions $u^{n}$ of (5.4) belong to $C^{1}(\bar{Q}) \cap C^{2}(Q)$ and $\psi^{n} \rightarrow \psi$ in $H^{1}(S)$. Then the estimate (5.5) holds for all differences $w:=u^{n}-u^{m}$ with a constant $c^{\prime}=c^{\prime}(R)$ (where $R$ is the Lipschitz constant of $f$ ), which is easily seen to be independent of $n$. Therefore, $\left(u_{n}\right)$ converges to a solution of (5.2), having the required regularity properties. 
Let us note that we also have the estimate

$$
\|u\|_{L^{\infty}(Q)} \leq c^{\prime \prime}
$$

with a constant $c^{\prime \prime}$ depending only on $\|\psi\|_{H^{1}(S)}$ and on the constant $\beta$ in the condition (1.5), but not on the Lipschitz constant of $f$. Indeed, applying the identity (5.3) for each $n$ (see the remark following the proof of the preceding proposition), we have

$$
\left\|u_{n}(0)\right\|_{L^{\infty}(-d, d)}^{2} \leq c E_{n}(0) \leq c+c \int_{Q}\left|F\left(u_{n}\right)\right| d x d t \leq c+c \int_{Q} L\left(u_{n}\right)^{2} d x d t \leq c+c \int_{0}^{d} L\left(\left\|u_{n}(t)\right\|_{L^{\infty}(t-d, d-t)}^{2}\right) d t
$$

Since the time 0 plays no special role here, we have more generally

$$
\left\|u_{n}(s)\right\|_{L^{\infty}(s-d, d-s)}^{2} \leq c+c \int_{s}^{d} L\left(\left\|u_{n}(t)\right\|_{L^{\infty}(t-d, d-t)}^{2}\right) d t
$$

for all $0 \leq s \leq t$, with the same constant $c$. Applying Theorem 2.1 we conclude that $\left\|u_{n}\right\|_{L^{\infty}(Q)}$ is bounded by some constant depending only on $\beta$ and on $\left\|\psi_{n}\right\|_{H^{1}(S)}$. Letting $n \rightarrow \infty$ we obtain the desired estimate (5.9).

Now let us turn to the case where $f$ is not globally Lipschitz continuous. Let us denote by $v^{n}$ the solution of (5.1) where $f$ is replaced by the globally Lipschitz continuous function $f_{n}$ defined by

$$
f_{n}(s):= \begin{cases}n & \text { if } f(s)>n \\ -n & \text { if } f(s)<-n \\ f(s) & \text { otherwise }\end{cases}
$$

By the preceding estimate (5.9) the sequence $\left(v^{n}\right)$ is bounded in $L^{\infty}(Q)$, because all functions $f_{n}$ satisfy the same growth conditions as $f$. It follows that if $n$ is larger than this common bound, then $v_{n}$ also solves (5.1) with the original $f$.

Turning to the proof of Theorem 1.3, let us set

$$
\begin{aligned}
A & =(a, 0), \\
B & =(a, T), \\
C & =(b, T), \\
D & =(b, 0), \\
E & =\left(\frac{a+b}{2}, \frac{b-a}{2}\right), \\
F & =\left(\frac{a+b}{2}, T-\frac{b-a}{2}\right) .
\end{aligned}
$$

Furthermore, let us denote by $K_{1}$ the triangle $A D E$, by $K_{2}$ the triangle $B F C$, by $K_{3}$ the trapezoid $A E F B$ and by $K_{4}$ the trapezoid $C F E D$; see Figure 2.

By Proposition 5.1 the Cauchy problem

$$
\left\{\begin{array}{l}
u_{t t}-u_{x x}-f(u)=0 \text { in } K_{1} \\
u(0, x)=u_{0}(x) \text { and } u_{t}(0, x)=u_{1}(x) \text { for } x \in(a, b)
\end{array}\right.
$$




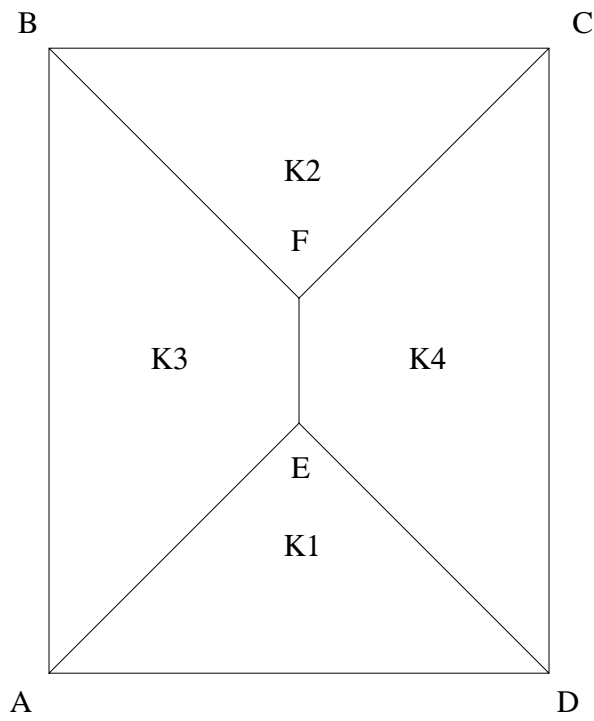

FiguRE 2

has a unique solution $u^{1}$, and its trace on the line segments $A E, D E$ belongs to $H^{1}(A E)$ and $H^{1}(D E)$, respectively.

Similarly, the Cauchy problem

$$
\left\{\begin{array}{l}
u_{t t}-u_{x x}-f(u)=0 \quad \text { in } K_{2}, \\
u(0, x)=v_{0}(x) \text { and } u_{t}(0, x)=v_{1}(x) \text { for } x \in(a, b)
\end{array}\right.
$$

has a unique solution $u^{2}$, and its trace on the line segments $B F, C F$ belongs to $H^{1}(B F)$ and $H^{1}(C F)$, respectively.

Now choose an arbitrary function $z_{0} \in H^{1}(E F)$ which has the same values at the endpoints $E$ and $F$ of the segment $E F$ as $u^{1}$ and $u^{2}$. Furthermore, fix a function $z_{1} \in L^{2}(E F)$ arbitrarily.

We are going to prove that the Goursat problem

$$
\left\{\begin{array}{l}
u_{t t}-u_{x x}-f(u)=0 \text { in } K_{3}, \\
u=u^{1} \text { on } A E, \\
u=z_{0} \text { on } E F, \\
u=u^{2} \text { on } B F, \\
\partial u / \partial x=z_{1} \text { on } E F
\end{array}\right.
$$


has a unique solution $u^{3}$, and similarly, that the Goursat problem

$$
\left\{\begin{array}{l}
u_{t t}-u_{x x}-f(u)=0 \text { in } K_{4}, \\
u=u^{1} \text { on } D E \\
u=z_{0} \text { on } E F \\
u=u^{2} \text { on } C F, \\
\partial u / \partial x=z_{1} \text { on } E F
\end{array}\right.
$$

has a unique solution $u^{4}$. By symmetry, we only consider the first one in the trapezoid $K_{3}$.

Let us denote by $G$ the intersection of the lines $A E$ and $B F$, and let us denote by $K_{5}$ the triangle $E G F$. Exchanging the role of the variables $t$ and $x$ and applying Proposition 5.1 again, we obtain that the problem

$$
\left\{\begin{array}{l}
u_{t t}-u_{x x}-f(u)=0 \text { in } K_{5}, \\
u=z_{0} \text { on } E F \\
\partial u / \partial x=z_{1} \text { on } E F
\end{array}\right.
$$

has a unique solution whose traces on the line segments $F G$ and $E G$ belong to $H^{1}(F G)$ and $H^{1}(E G)$, respectively.

Now consider the usual Goursat problem

$$
\left\{\begin{array}{l}
u_{t t}-u_{x x}-f(u)=0 \text { in } K_{6}, \\
u=\varphi \text { on } S,
\end{array}\right.
$$

where $K_{6}$ denotes the triangle $A G B, S$ denotes the union of the four line segments $B F, F G, G E$ and $E A$, and $\varphi$ is given by the restrictions to these four line segments of the solutions of the above three Cauchy problems in the triangles $K_{1}, K_{2}$ and $K_{5}$. By Proposition 5.1 we have $\varphi \in H^{1}(S)$. Applying Proposition 5.2 (we exchange again the sense of the space and time variables), the problem (5.11) has a unique solution, and its trace on the side $A B$ belongs to $H^{1}(A B)$.

In order to prove uniqueness, let $u_{1}$ and $u_{2}$ be two solutions of (5.10). Then the above method allows us to extend them to two solutions $\tilde{u}_{1}$ and $\tilde{u}_{2}$ of (5.11) with the same boundary data $\varphi$. We conclude by using the uniqueness part of Proposition 5.2 that $\tilde{u}_{1} \equiv \tilde{u}_{2}$ and thus $u_{1} \equiv u_{2}$.

Finally, we claim that the formula

$$
u:=u^{i} \quad \text { in } \quad K_{i}(i=1, \ldots, 4)
$$

defines a solution of (1.7) with $h_{a}, h_{b} \in H^{1}(0, T)$ given by the traces of $u$, and satisfying the desired final conditions by construction. The only property to verify is that the differential equation in (1.7) is satisfied in the whole rectangle $R:=(a, b) \times(0, T)$ and not only in the subdomains $K_{1}, K_{2}, K_{3}$ and $K_{4}$. More precisely, we have to prove the

Lemma 5.3. The equation

$$
\int_{R} u\left(\partial_{t}^{2}-\partial_{x}^{2}\right) \varphi d x d t=\int_{R} f(u) \varphi d x d t
$$

is satisfied for all $\varphi \in C_{0}^{\infty}(R)$.

The proof of this lemma involves nothing else than the theory of linear hyperbolic systems. For the reader's convenience we give a proof in an Appendix. 


\section{Appendix. Proof of lemma 5.3}

We proceed in two steps.

Lemma 6.1. The equation (5.12) is satisfied for all $\varphi \in C_{0}^{\infty}\left(R^{\prime}\right)$ where $R^{\prime}=R-\{E, F\}$.

Proof. Set

$$
S_{i j}=\overline{K_{i}} \cap \overline{K_{j}}, \quad 1 \leq i \neq j \leq 4
$$

and

$$
S=\cup_{i \neq j} S_{i j}
$$

Let us fix $\varphi \in C_{0}^{\infty}\left(R^{\prime}\right)$. We can cover supp $\varphi$ with a finite collection $B_{1}, \ldots, B_{N}$ of open balls contained in $R$ such that

$$
E, F \notin \cup_{k=1}^{N} \overline{B_{k}},
$$

and that each $\overline{B_{k}}$ intersects at most one of the sets $S_{i j}$.

Let $1=\sum_{k=1}^{N} \psi_{k}$ be a partition of unity related to such a collection. Then

$$
\varphi=\sum_{k=1}^{N} \varphi_{k}, \quad \varphi_{k}=\varphi \psi_{k}, \quad \operatorname{supp} \varphi_{k} \subset \overline{B_{k}}
$$

It suffices to show that

$$
\int_{R} u\left(\partial_{t}^{2}-\partial_{x}^{2}\right) \varphi_{k} d x d t=\int_{R} f(u) \varphi_{k} d x d t, \quad k=1, \ldots, N
$$

If $\overline{B_{k}} \cap S=\emptyset$, this equality follows directly from the assumptions. Let us assume that $\overline{B_{k}} \cap S \neq \emptyset$, then $\overline{B_{k}} \cap S=\overline{B_{k}} \cap S_{i j}$ for some $i, j$.

If $S_{i j}=S_{34}$, then the equality follows because $u_{3}$ and $u_{4}$ agree on $S_{34}$ together with their normal derivatives.

The remaining four cases are similar: suppose, for instance, that $S_{i j}=S_{13}$. Then

$$
\int_{R} u\left(\partial_{t}^{2}-\partial_{x}^{2}\right) \varphi_{k} d x d t=\left(\int_{B_{k} \cap K_{1}}+\int_{B_{k} \cap K_{3}}\right) u\left(\partial_{t}^{2}-\partial_{x}^{2}\right) \varphi_{k} d x d t
$$

Moreover, using the normal vector $\nu=(1,-1)$ on $S_{13}$,

$$
\begin{aligned}
\int_{B_{k} \cap K_{1}} u\left(\partial_{t}^{2}-\partial_{x}^{2}\right) \varphi_{k} d x d t= & \int_{B_{k} \cap K_{1}}\left(\partial_{t}-\partial_{x}\right)\left[u\left(\partial_{t}+\partial_{x}\right) \varphi_{k}\right]-\left(\partial_{t}-\partial_{x}\right) u\left(\partial_{t}+\partial_{x}\right) \varphi_{k} d x d t \\
= & \int_{B_{k} \cap S_{13}} u\left(\partial_{t}+\partial_{x}\right) \varphi_{k}\left(\nu_{t}-\nu_{x}\right) d S-\int_{B_{k} \cap K_{1}}\left(\partial_{t}+\partial_{x}\right) \varphi_{k}\left(\partial_{t}-\partial_{x}\right) u d x d t \\
= & 2 \int_{B_{k} \cap S_{13}} u\left(\partial_{t}+\partial_{x}\right) \varphi_{k} d S-\int_{B_{k} \cap S_{13}} \varphi_{k}\left(\partial_{t}-\partial_{x}\right) u\left(\nu_{t}+\nu_{x}\right) d S \\
& +\int_{B_{k} \cap K_{1}} \varphi_{k} f(u) d x d t
\end{aligned}
$$

Since $\nu_{t}+\nu_{x}=0$ on $S_{13}$, we conclude that

$$
\int_{B_{k} \cap K_{1}} u\left(\partial_{t}^{2}-\partial_{x}^{2}\right) \varphi_{k} d x d t=2 \int_{B_{k} \cap S_{13}} u\left(\partial_{t}+\partial_{x}\right) \varphi_{k} d S+\int_{B_{k} \cap K_{1}} \varphi_{k} f(u) d x d t
$$


Similarly,

$$
\begin{aligned}
\int_{B_{k} \cap K_{3}} u\left(\partial_{t}^{2}-\partial_{x}^{2}\right) \varphi_{k} d x d t= & \int_{B_{k} \cap S_{13}} u\left(\partial_{t}+\partial_{x}\right) \varphi_{k}\left(\nu_{x}-\nu_{t}\right) d S-\int_{B_{k} \cap S_{13}} \varphi_{k}\left(\partial_{t}-\partial_{x}\right) u\left(-\nu_{t}-\nu_{x}\right) d S \\
& +\int_{B_{k} \cap K_{3}} \varphi_{k} f(u) d x d t ;
\end{aligned}
$$

since $\nu_{x}-\nu_{t}=-2$ and $-\nu_{t}-\nu_{x}=0$ on $S_{13}$, we conclude that

$$
\int_{B_{k} \cap K_{3}} u\left(\partial_{t}^{2}-\partial_{x}^{2}\right) \varphi_{k} d x d t=-2 \int_{B_{k} \cap S_{13}} u\left(\partial_{t}+\partial_{x}\right) \varphi_{k} d S+\int_{B_{k} \cap K_{3}} \varphi_{k} f(u) d x d t .
$$

Adding together the equalities (6.2) and (6.3), the boundary terms cancel each other and we obtain (6.1).

The same computation works if $S_{i j}=S_{24}$. If $S_{i j}=S_{14}$ or $S_{i j}=S_{23}$, then we can similarly argue, exchanging the roles of the operators $\partial_{t}-\partial_{x}$ and $\partial_{t}+\partial_{x}$.

The twofold application of following lemma will complete the proof of Lemma 5.3. First we apply it with $g=f(u), \Omega=R-\{E\}$ and $P=F$, and then with $g=f(u), \Omega=R$ and $P=E$.

Lemma 6.2. Let $\Omega$ be an open set in $\mathbb{R}^{2}$ and let $P \in \Omega$. If for some $u \in H^{1}(\Omega)$ and $g \in L^{2}(\Omega)$ the equality

$$
\int_{\Omega} u\left(\partial_{t}^{2}-\partial_{x}^{2}\right) \varphi d x d t=\int_{\Omega} g \varphi d x d t
$$

is satisfied for all $\varphi \in C_{0}^{\infty}(\Omega-\{P\})$, then it is also satisfied for all $\varphi \in C_{0}^{\infty}(\Omega)$.

Proof. Since the points have zero capacity in $\mathbb{R}^{2}$, there exists for every $\varepsilon>0$ a function $\varphi_{\varepsilon} \in C_{0}^{\infty}\left(B_{\varepsilon}(P)\right)$ and a number $0<\rho_{\varepsilon}<\varepsilon$ such that

$$
0 \leq \varphi_{\varepsilon}(x, t) \leq 1, \quad \varphi_{\varepsilon}(x, t)=1 \quad \text { if } \quad|(x, t)-P| \leq \rho_{\varepsilon}, \quad \int_{B_{\varepsilon}(P)}\left|\nabla \varphi_{\varepsilon}\right|^{2} d x d t \leq \varepsilon
$$

(See, e.g., Maz'ja [7] for proof.)

Let $\varphi \in C_{0}^{\infty}(\Omega)$, then

$$
\varphi=\varphi \varphi_{\varepsilon}+\varphi\left(1-\varphi_{\varepsilon}\right)=: \varphi_{\varepsilon}^{1}+\varphi_{\varepsilon}^{2}
$$

with

$$
\varphi_{\varepsilon}^{2} \in C_{0}^{\infty}(\Omega-\{P\})
$$

Therefore

$$
\begin{aligned}
\int_{\Omega} u\left(\partial_{t}^{2}-\partial_{x}^{2}\right) \varphi d x d t & =\int_{\Omega} u\left(\partial_{t}^{2}-\partial_{x}^{2}\right) \varphi_{\varepsilon}^{1} d x d t+\int_{\Omega} u\left(\partial_{t}^{2}-\partial_{x}^{2}\right) \varphi_{\varepsilon}^{2} d x d t \\
& =-\int_{\Omega}\left(\partial_{t} u \partial_{t} \varphi_{\varepsilon}^{1}-\partial_{x} u \partial_{x} \varphi_{\varepsilon}^{1}\right) d x d t+\int_{\Omega} g \varphi_{\varepsilon}^{2} d x d t
\end{aligned}
$$

Now, notice that

$$
\left\|\varphi-\varphi_{\varepsilon}^{2}\right\|_{L^{2}(\Omega)}=\left\|\varphi \varphi_{\varepsilon}\right\|_{L^{2}\left(B_{\varepsilon}(P)\right)} \rightarrow 0
$$


as $\varepsilon \rightarrow 0$, and that

$$
\left|\int_{\Omega}\left(\partial_{t} u \partial_{t} \varphi_{\varepsilon}^{1}-\partial_{x} u \partial_{x} \varphi_{\varepsilon}^{1}\right) d x d t\right| \leq\|\nabla u\|_{L^{2}(\Omega)}\left\|\nabla \varphi_{\varepsilon}^{1}\right\|_{L^{2}(\Omega)}
$$

Using the choice of $\varphi_{\varepsilon}$ we have

$$
\left\|\nabla \varphi_{\varepsilon}^{1}\right\|_{L^{2}(\Omega)} \leq\|\nabla \varphi\|_{L^{2}(\Omega)}\left\|\varphi_{\varepsilon}\right\|_{L^{2}\left(B_{\varepsilon}(P)\right)}+\|\varphi\|_{L^{2}(\Omega)}\left\|\nabla \varphi_{\varepsilon}\right\|_{L^{2}\left(B_{\varepsilon}(P)\right)} \rightarrow 0
$$

as $\varepsilon \rightarrow 0$, and so

$$
\left|\int_{\Omega} u\left(\partial_{t}^{2}-\partial_{x}^{2}\right) \varphi d x d t-\int_{\Omega} g \varphi d x d t\right| \leq\|g\|_{L^{2}(\Omega)}\left\|\varphi-\varphi_{\varepsilon}^{2}\right\|_{L^{2}(\Omega)}+\|\nabla u\|_{L^{2}(\Omega)}\left\|\nabla \varphi_{\varepsilon}^{1}\right\|_{L^{2}(\Omega)} \rightarrow 0 .
$$

Hence the lemma follows.

\section{REFERENCES}

[1] T. Cazenave and A. Haraux, Équations d'évolution avec non linéarité logarithmique. Ann. Fac. Sci. Toulouse 2 (1980) $21-51$.

[2] T. Cazenave and A. Haraux, Introduction aux problèmes d'évolution semi-linéaires. Mathématiques et applications, Vol. 1, Ellipses et SMAI, Paris (1990).

[3] P. Erdős, On the law of the iterated logarithm. Ann. of Math. 43 (1942) 419-436.

[4] O.Yu. Imanuvilov, Boundary control of semilinear evolution equations. Russian Math. Surveys 44 (1989) 183-184.

[5] Li Ta-Tsien and Bing-Yu Zhang, Global exact controllability of a class of quasilinear hyperbolic systems. J. Math. Anal. Appl. 225 (1998) 289-311.

[6] J.-L. Lions, Quelques méthodes de résolution des problèmes aux limites non linéaires. Dunod-Gauthier-Villars, Paris (1969).

[7] V.G. Maz'ja, Sobolev Spaces. Springer-Verlag, New York (1985).

[8] A. Pazy, Semigroups of Linear Operators and Applications to Partial Differential Equations. Springer-Verlag, New York (1983).

[9] S.L. Sobolev, Partial Differential Equations of Mathematical Physics. Dover, New York (1989).

[10] E. Zuazua, Exact controllability for semilinear wave equations in one space dimension. Ann. Inst. H. Poincaré Anal. Non Linéaire 10 (1993) 109-129. 